\title{
Observation of accelerated beam ion population during edge localized modes in the ASDEX Upgrade tokamak
}

Galdon-Quiroga, J.; Garcia-Munoz, M.; McClements, K. G.; Nocente, M.; Denk, S. S.; Freethy, S.; Jacobsen, A. S; Orain, F.; Rivero-Rodriguez, J. F.; Salewski, M.

Total number of authors:

16

Published in:

Nuclear Fusion

Link to article, DOI:

10.1088/1741-4326/ab1376

Publication date:

2019

Document Version

Peer reviewed version

Link back to DTU Orbit

Citation (APA):

Galdon-Quiroga, J., Garcia-Munoz, M., McClements, K. G., Nocente, M., Denk, S. S., Freethy, S., Jacobsen, A. S., Orain, F., Rivero-Rodriguez, J. F., Salewski, M., Sanchis-Sanchez, L., Suttrop, W., Viezzer, E., Willensdorfer, M., ASDEX Upgrade Team, \& EUROfusion MST1 Team (2019). Observation of accelerated beam ion population during edge localized modes in the ASDEX Upgrade tokamak. Nuclear Fusion, 59, [066016]. https://doi.org/10.1088/1741-4326/ab1376

\section{General rights}

Copyright and moral rights for the publications made accessible in the public portal are retained by the authors and/or other copyright owners and it is a condition of accessing publications that users recognise and abide by the legal requirements associated with these rights.

- Users may download and print one copy of any publication from the public portal for the purpose of private study or research.

- You may not further distribute the material or use it for any profit-making activity or commercial gain

- You may freely distribute the URL identifying the publication in the public portal 


\title{
Observation of accelerated beam ion population during edge localized modes in the ASDEX Upgrade tokamak
}

\author{
J.Galdon-Quiroga ${ }^{1,2}$, M.Garcia-Munoz ${ }^{2,3}$, \\ K.G.McClements ${ }^{4}$, M.Nocente ${ }^{5}$, S.S.Denk ${ }^{1}$, S.Freethy ${ }^{1}$, \\ A.S.Jacobsen ${ }^{1}$, F.Orain ${ }^{1}$, J.F.Rivero-Rodriguez ${ }^{3,6}$, \\ M.Salewski ${ }^{7}$, L.Sanchis-Sanchez ${ }^{2,3}$, W.Suttrop ${ }^{1}$, \\ E.Viezzer ${ }^{2,3}$, M.Willensdorfer ${ }^{1}$, the ASDEX Upgrade Team \\ and the EUROfusion MST1 Team $\ddagger$ \\ 1 Max Planck Institute for Plasma Physics, Boltzmannstr. 2, 85748 Garching, \\ Germany \\ 2 Dept. of Atomic, Molecular and Nuclear Physics, University of Seville, Avda. \\ Reina Mercedes, 41012 Seville, Spain \\ 3 Centro Nacional de Aceleradores CNA (Universidad de Sevilla, Junta de \\ Andalucia, CSIC), Avda. Thomas A. Edison 7, 41092 Seville, Spain \\ 4 CCFE, Culham Science Centre, Abingdon OX14 3DB, United Kingdom \\ 5 Dipartimento di Fisica G Occhialini, Universita di Milano-Bicocca, Milano, \\ Italy \\ 6 Department of Mechanical Engineering and Manufacturing, University of \\ Seville, Camino de los Descubrimientos s/n, 41092, Seville, Spain \\ 7 Department of Physics, Technical University of Denmark, Kgs. Lyngby, \\ Denmark \\ E-mail: joaquin.galdon@ipp.mpg.de jgaldon@us.es
}

\begin{abstract}
.
The interaction between fast-ions and edge localized modes (ELMs) is investigated by means of fast-ion loss detector (FILD) measurements. Fast-ion losses are increased during ELMs exhibiting a 3D filamentary-like behaviour. An accelerated beam ion population has been observed during ELMs in a tokamak for the first time. Tomographic inversion of the measured fast-ion losses reveal multiple velocity-space structures. Attending to the experimental observations, an acceleration mechanism is proposed based on a resonant interaction between the beam ions and parallel electric fields emerging during the ELM crash. The key experimental observations can be qualitatively reproduced by full-orbit following simulations of fast-ions in the presence of the ELM magnetic and electric perturbation fields. Our findings may shed light on the possible contribution of fast-ions to the ELM stability and the transient heat loads on plasma facing components.
\end{abstract}

$\ddagger$ See the author list of H. Meyer et al., Nucl. Fusion 57102014 (2017). 


\section{Introduction}

Edge localized modes (ELMs)[1] are closely linked to the high confinement mode (H-mode)[2] in fusion devices, which is the foreseen operational regime for future burning plasma experiments [3]. ELMs are cyclic magnetohydrodynamic (MHD) instabilities driven by the current and pressure gradient at the edge of tokamak plasmas $[3,4]$. During an ELM, the pedestal profiles collapse, leading to a sudden loss of confinement at the plasma edge. Energy and particles are released to the machine first wall, thus potentially limiting the lifetime of plasma facing components in future devices like ITER [5]. It is therefore important to investigate the effect of ELMs on the confinement of fast-ions and how can these contribute to the transient heat loads delivered to plasma facing components

In this paper we present time resolved velocityspace measurements of ELM induced fast-ion losses in the ASDEX Upgrade (AUG) tokamak. The measurements have been performed with scintillator based fast-ion loss detectors (FILD)[6] located at different toroidal positions above the AUG midplane $(z \sim 0.3 \mathrm{~m})$. Intra-ELM velocity space measurements of fast-ion losses reveal a population at energies well above the main NBI injection energy, well correlated in time with the NBI systems and the occurrence of ELMs. This observation is interpreted as the acceleration of beam ions in the presence of parallel electric fields arising during ELMs. Bursts in the electron cyclotron emission and soft X-ray diagnostics are also observed at the onset of these ELMs [7], similar to previous observations in MAST, which are indicative of electron acceleration [8], thus supporting the hypothesis of the presence of electric fields. Although previous investigations have reported ion acceleration in magnetically confined plasmas during internal reconnection events $[9,10]$ and mergingcompression during plasma start-up [11], this is the first experimental indication of ion acceleration during ELMs in a tokamak. Furthermore, particle acceleration is ubiquitous in space and astrophysical plasmas and measurements provided by laboratory experiments like these may help to shed light on the fundamental physics underlying these phenomena [12]. The detailed experimental observations suggest that the mechanism responsible for the ion acceleration is a resonant interaction between the beam ion orbits and the parallel electric fields associated with the ELMs. To investigate the fast-ion transport induced by ELMs, full-orbit following simulations have been carried out in the presence of the ELM perturbed magnetic configuration calculated with the JOREK code [13]. In order to assess the viability of the proposed acceleration mechanism, additional simulations have been carried out including a parametrized model which
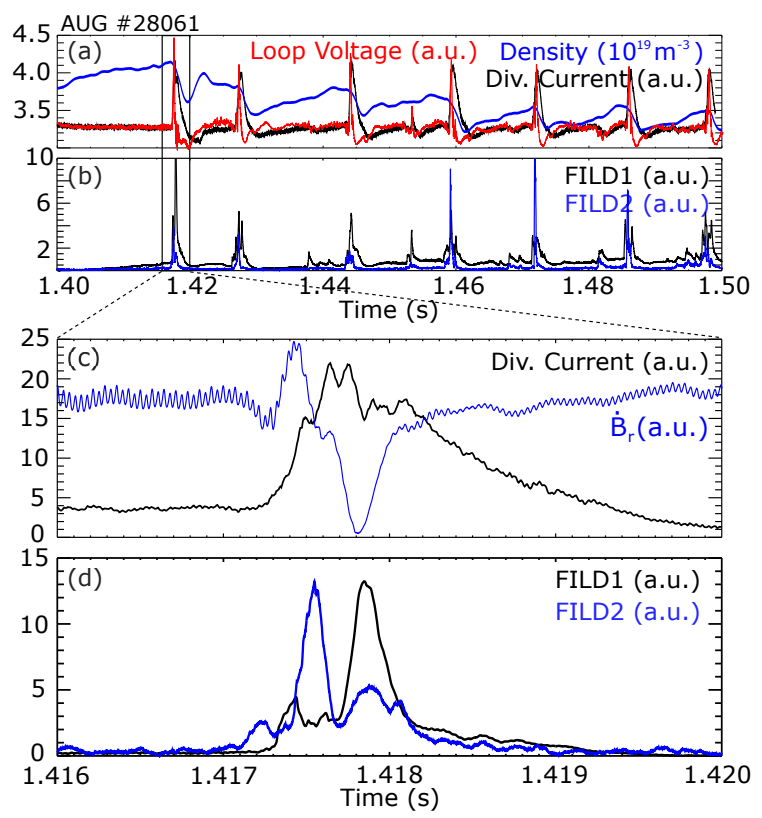

Figure 1. (a) Timetraces of the electron density (blue), the loop voltage (red) and the divertor current (black), which is used as an ELM monitor. (b) Timetraces of two different FILD detectors, located at the same poloidal position but toroidally displaced. (c) and (d) show a zoom into a single ELM.

resembles the 3D character of the parallel electric fields associated with the ELM.

The paper is organized as follows: in Sec.2 the experimental observations are described, including time resolved velocity space measurements of fastion losses; in Sec.3 simulations of fast-ions in the presence of magnetic and electric field perturbations are presented. Finally, a summary is presented and the conclusions are discussed in Sec.4.

\section{Experimental measurements}

The experiments have been performed in deuterium plasmas with a low density $\left(n_{e} \leq 6 \cdot 10^{19} \mathrm{~m}^{-3}\right)$, a low pedestal collisionality $\left(\nu^{*} \leq 0.4\right)$ and a normalized plasma-to-magnetic pressure ratio of $\beta_{N}=\beta \frac{a B_{t}}{I_{p}} \sim 2.5$, where $\beta$ is the volume averaged toroidal plasma-tomagnetic pressure ratio (in \%), $a$ is the minor radius of the tokamak (in $\mathrm{m}$ ), $B_{t}$ is the toroidal magnetic field (in T) and $I_{p}$ the plasma current (in MA). The experiments were performed in a variety of scenarios with plasma current ranging from $0.6-1.0 \mathrm{MA}$ and toroidal magnetic field ranging from $1.8-2.5 \mathrm{~T}$, in which the only external sources of fast-ions to the plasma were the neutral beam injection (NBI) systems, in the absence of ion cyclotron resonance heating (ICRH).

The main diagnostic used in this study has been a scintillator based fast-ion loss detector (FILD). The 
FILD work as a magnetic spectrometer, making use of the tomakak magnetic to disperse ions into a scintillator plate. The velocity-space of the escaping fast-ion distribution (gyroradius and pitch angle) can be inferred from the strike position of the ions on the scintillator. In AUG, the light emmitted by the scintillator plate is imaged simultaneously by two systems: a fast acquisition system consisting on a set of photomultiplier tubes (PMTs), which provide a time resolution of $1 \mathrm{MHz}$ but poor spatial resolution; and a camera, which provides good spatial resolution for the identification of the velocity-space of the lost ions, but at lower temporal resolution. Although multiple FILD detectors are available at AUG, in this study we focus on the measurements carried out with FILD1. The reason is that a fast CMOS camera was installed in the FILD1 setup, which allowed us to improve the temporal resolution down to $\sim 1 \mathrm{kHz}$ frame rate, beyond the $50 \mathrm{~Hz}$ of the standard CCD cameras used in the other FILD systems.

Fig.1(a) shows timetraces of the density (blue), the divertor current, which is used as an ELM monitor in AUG (black), and the loop voltage (red). Fig.1(b) shows the signal of two different FILD detectors which are located at the same poloidal position $(z \sim 0.3$ m) but at different toroidal positions. Different ELM crashes with typical timescales of $\sim 1 \mathrm{~ms}$ can be observed during which the divertor current increases rapidly, the density collapses and the fast-ion losses measured by both FILD systems increase. A zoom into an individual ELM crash is shown in Figs.1(c) and (d), where the timetrace of the associated magnetic perturbation is also plotted. The first thing to notice is the filamentary-like behaviour of the FILD signals, in which multiple spikes with characteristic times on the order of $\sim 100 \mu$ s are observed within a single ELM crash. The second thing to notice is that the time evolution of these fast-ion filaments is different for the two FILD detectors, thus revealing the 3D nature of the phenomenon. The deeper deposition in the NBI birth profile due to lower densities during the ELM crash [14] suggests that the increased fast-ion losses are likely to be produced by the perturbation fields associated with the ELM.

\subsection{Velocity space of ELM induced fast-ion losses}

FILD detectors provide time-resolved velocity space measurements of fast-ion losses during the ELM crash. An example of this is shown in Fig.2, which corresponds to AUG FILD1 data during shot \#33127 at $4.354 \mathrm{~s}$. Here a set of two populations can be observed at two main different pitch angles of $\sim$ $45^{\circ}$ and $\sim 60^{\circ}$, where the pitch angle is defined as $\arccos \left(-\frac{v_{\|}}{v}\right)$. These two populations correspond to first orbit losses of NBI sources $Q 7$ and $Q 8$ respectively.
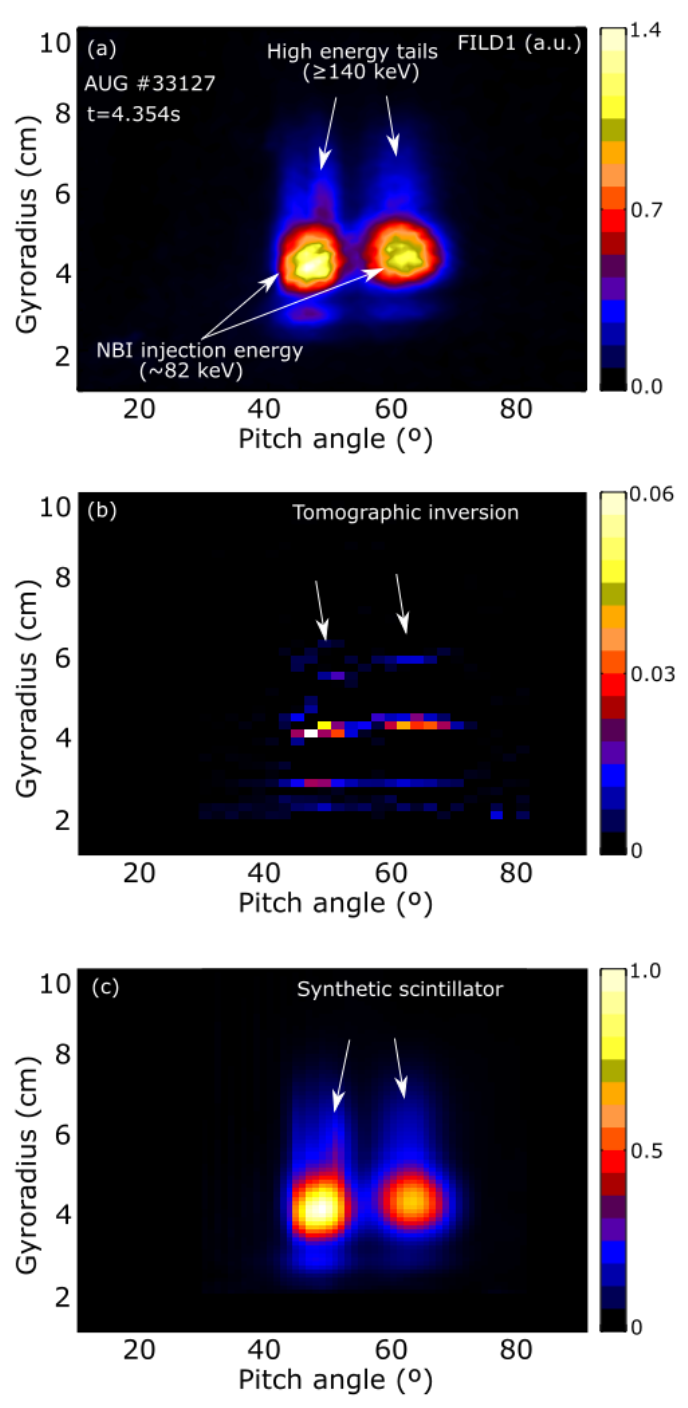

Figure 2. (a) Velocity space measurement of fast-ion losses performed with FILD1 during an ELM in AUG \#33127. (b) Undistorted velocity space of the fast-ion losses reaching the detector pinhole, as a result of the application of the tomographic inversion. (c) Synthetic scintillator signal retrieved from the tomography. The location of the high-energy feature is indicated by white arrows.

A clear pattern is observed in the gyroradii of both populations. At AUG, the NBI injects neutrals at three different energies $E_{0}, E_{0} / 2$ and $E_{0} / 3$, due to the presence of molecular deuterium $D_{1}^{+}, D_{2}^{+}$and $D_{3}^{+}$in the sources. These different energy components can be seen in the FILD signal: a main spot centered at $r_{L} \sim$ $4 \mathrm{~cm}$, which corresponds to the main NBI injection energy $E_{0}=82 \mathrm{keV}$; a second spot centered at $r_{L} \sim 3$ $\mathrm{cm}$, corresponding to the half energy component of the NBI. Additionally, a population at $r_{L} \geq 5 \mathrm{~cm}$, which corresponds to energies well above the main NBI injection and is only observed during the transient 


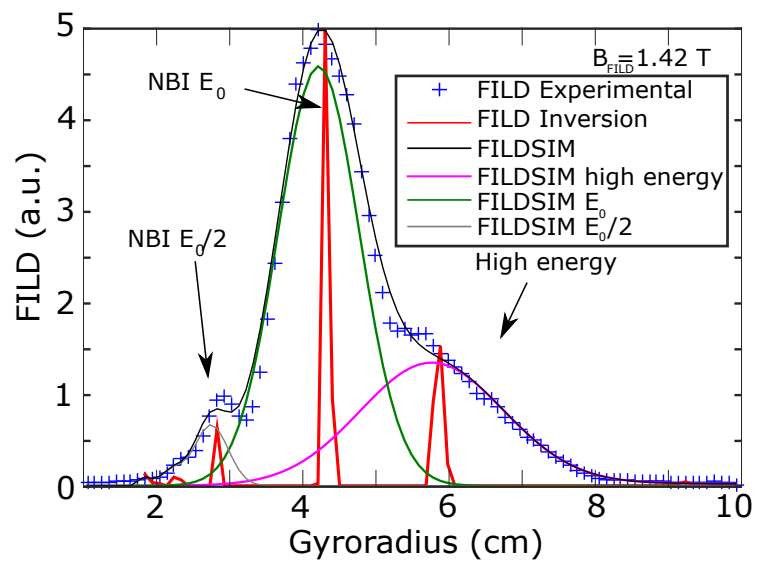

Figure 3. Gyroradius profile of the FILD intensity integrated in pitch angle between $58^{\circ}$ and $65^{\circ}$. The blue crosses are the experimental measurements. The result of the tomographic inversion is shown in red. The recovered synthetic FILD signal is shown in black. The different colors correspond to the contribution of the different fast-ion populations: $E_{0}$ in green, $E_{0} / 2$ in grey and the high-energy feature in magenta.

ELM crash. This population shall be referred to as the high-energy feature in the rest of the paper.

The signal shown in Fig.2(a) is directly obtained from the light pattern measured in the FILD scintillator. The finite size for the different spots is due to the finite resolution of the system in velocity space [15]. The resolution in pitch angle is mainly determined by the length of the detector pinhole and is on the order of $\sim 1^{\circ}-2^{\circ}$. However, the resolution in gyroradius (energy) decreases as the gyroradius of the measured ions increases. This is due to the fact that, as the gyroradius increases, the orbit becomes more like a straight line within the size of the probe head, and therefore the collimator loses its capability to block orbits.

The measurement of the velocity space of fastion losses at the scintillator can then be understood as a distortion of the velocity space of fast-ions reaching the detector pinhole, which is the quantity that we aim to measure. In order to overcome this limitation, the FILDSIM code has been developed which models the response of the detector based on a weight function formalism, similar to those used for other fast-ion diagnostics such as FIDA or CTS $[16,17]$.For a more comprehensive description of the response of scintillator based FILDs, we refere the reader to [15]. The undistorted velocity space distribution of the lost fast-ions reaching the detector pinhole can then be retrieved by the application of velocity-space tomographic techniques $[18,19,20]$. In the case of FILD signals the $0^{\text {th }}$ order Tikhonov regularization method has been applied including a non-negativity constraint, which is trivially justified for the measurement of fast-ion distributions [20]. The result of this analysis is shown in Fig.2(b). It can be observed that the fast-ion populations giving rise to the main spots (NBI first orbit losses) in Fig.2(a) are well localized at the corresponding NBI injection energy. Furthermore, the population corresponding to the high-energy feature is surprisingly also well-localized in energy, rather than being an energy spread. The synthetic FILD signal retrieved from the tomographic inverted distribution is shown in Fig.2(c) which is in good agreement with the experimental measurement shown in Fig.2(a). A more detailed comparison is shown in Fig.3, where the gyroradius profile of the FILD signal integrated along the pitch angle interval between $58^{\circ}$ and $65^{\circ}$ is plotted. The blue crosses represent the experimental signal, which is smoothed due to the effect of the instrument resolution. The result of the tomographic inversion is plotted in red, where three peaks well defined in energy can be observed at $r_{L}=4.1 \mathrm{~cm}$ (corresponding to the main NBI injection energy $\left.E_{0}=82 \mathrm{keV}\right)$, at $r_{L}=2.9 \mathrm{~cm}$ (corresponding to $E_{0} / 2=41 \mathrm{keV}$ ) and at $r_{L}=5.8$ $\mathrm{cm}$, which corresponds to an energy of $\sim 160 \mathrm{keV}$. The synthetic signal is plotted in black, showing a good agreement with the experimental signal. The contributions to the synthetic signal from the different populations are plotted in magenta (for the highenergy feature), in green (for $E_{0}$ ) and grey (for $E_{0} / 2$ ).

\subsection{Correlation of high-energy population with ELMs}

The observation of the high-energy feature is reproducible and well correlated with the NBI heating systems and the occurrence of ELMs. This is illustrated in Fig.4(a), where the evolution in time of the gyroradius profile measured by FILD is represented. In this case, since we are only interested in investigating the temporal correlation of the high-energy feature with ELMs and the onset of the NBI systems, the analysis has been carried out using the non-inverted FILD signals, due to the large computation times needed for these tomographic inversions. The divertor current is plotted in white and the NBI power of the source contributing to the FILD signal is plotted in red. Before the NBI source is switched on, there is no signal in the FILD, despite an ELM occurring at $1.173 \mathrm{~s}$. At $1.20 \mathrm{~s}$ the NBI is switched on and the FILD starts to measure the main and half NBI energy components, which are centered at $r_{L}=4.1 \mathrm{~cm}$ and $r_{L}=2.9 \mathrm{~cm}$ respectively. Then, everytime an ELM crash takes place, indicated by the spikes in the divertor current signal, the high-energy feature appears in FILD, as it can be seen in the four ELMs taking place between 1.2 and $1.28 \mathrm{~s}$. The difference in the gyroradii profiles between inter-ELM periods and during ELMs is illustrated in Fig.4(b), where the FILD signals have been normalized to the unity in order to account for the difference 
in the intensity of the losses. The high-energy feature can be clearly observed during the ELM crash at 1.209 $\mathrm{s}$ (blue), where the FILD signal at $r_{L}>5 \mathrm{~cm}$ is above the level shown in the inter-ELM phases at $1.199 \mathrm{~s}$ and $1.220 \mathrm{~s}$ (black and red). The FILD signal showing the complete velocity space of the fast-ion losses before, during and after the ELM crash is shown in Fig.4(c), (d) and (e) respectively. It can be seen that the pitch angle distribution of the losses at the main and half energy injection components remains invariant during the ELM cycle.

Further evidence of the correlation between the high-energy feature measured by FILD and ELMs was found during operation in an ELM suppressed regime. In AUG ELM suppression can be achieved by the application of external magnetic perturbations (MPs) $[21,22,23]$ for certain values of $q_{95}$, which is the safety factor at $95 \%$ of the plasma minor radius, collisionality and differential phasing between the upper and lower sets of MP coils installed in AUG. Fig.5(a) shows the evolution of the FILD gyroradius profile measured during a whole shot in which MPs were applied, as indicated by the green box at the bottom of the figure. The divertor current is again plotted in white and used as an ELM monitor. Before the MP coils are switched on at $1.5 \mathrm{~s}$, the high-energy feature can be seen in the FILD. At $1.5 \mathrm{~s}$, the MPs are switched on, leading to an increased ELM frequency and decreased amplitude. The high-energy feature can still be observed during this ELM mitigated regime. However, in the time interval betwen 2.5 and $5.2 \mathrm{~s}$ the ELM suppressed regime is established and the highenergy feature dissappears from the FILD signal. At $5.2 \mathrm{~s}$ the ELM mitigated regime is recovered and hence the high-energy feature reappears in the FILD signal during the ELM crashes. The complete velocity space measured by FILD is shown in Fig.5(b) and (c) before and during the ELM suppressed phase respectively.

\subsection{Variation of the pitch angle structure with $q_{95}$}

While the pitch angle of the fast-ion losses at the main, half and third NBI energy components is not observed to change, it was found that the highenergy feature exhibits a pitch angle structure that depends on the beam source and $q_{95}$. Scans in $q_{95}$ were performed by ramping the toroidal magnetic field while keeping the plasma current constant. This is illustrated in Fig.6(a) which shows the pitch angle profile of the FILD signal for the main NBI injection energy (in black, for reference) and for the high-energy component (in colors). The different colors correspond to different time points (and therefore different $q_{95}$ values) indicated in Fig.6(b). The profile at the main NBI injection energy shows two bumps at $45^{\circ}$ and $60^{\circ}$ corresponding to first orbit losses of beam sources
Q7 and Q8 respectively. For the pitch angle interval between $40^{\circ}-50^{\circ}$ corresponding to Q7 (passing orbits), two different spikes labelled as I and II can be observed. In order to observe the relative changes in the FILD intensity between these two spikes the FILD signal has been normalized at each time point. In the first time point (blue curve), the intensity of spike II is larger than that of spike I. However, as $q_{95}$ is decreased during the discharge, the relative intensity of spike I increases with respect to spike II. In the second time point (red curve) the relative intensities are the same, while for the third and fourth time points (yellow and green curves) the relative intensity of spike I is larger than that of spike II. During the whole scan, however, no clear change in the pitch angle structure of the signals corresponding to beam ions from source Q8 (trapped particles) could be observed. Fig.6(c) and (d) show an additional scan in which $q_{95}$ was ramped from 4.7 to 5.4. In this case, no clear dependence of the pitch angle structure was observed.

\subsection{Electron cyclotron emission and soft $X$-ray bursts}

In addition to the measurement of fast-ion losses, spikes in soft X-ray emission [24] and bursts in electron cyclotron emission (ECE) [25] signals have also been observed at the onset of some of these ELMs [7]. This is shown in Fig.7, where (a) shows the timetrace of the divertor current and the FILD signal during an ELM. Fig.7 shows the signal of the ECE diagnostic measured in the position indicated by the black circle in Fig.7(d), assuming that the emission corresponds to the local second electron cyclotron harmonic. Large spikes can be observed at around $3.1825 \mathrm{~s}$. These bursts are localized in a small number of ECE channels indicating that the emission is constrained to a narrow frequency band, in this case between 110 and 112 GHz. The emission is likely produced by the buildup of a non-thermal electron population. Under this assumption, radiation transport modelling including broadening effects [26] has been carried out for the interpretation of these measurements, suggesting that the emission is originating at $\rho_{\text {pol }}>0.92$ by electrons with energies below $25 \mathrm{keV}$. In Fig.7(c) the signals of three different soft X-ray channels are shown. Their lines of sight near the edge of the plasma are indicated in Fig.7(d). The signals associated with the blue and green lines of sight, which are in the scrape-off layer and tangential to the separatrix, show bursts slightly after the ECE, while such behaviour is not observed for the line of sight passing through the separatrix. A similar observation of bursts in both the ECE and soft X-ray diagnostics was reported in MAST [8] to be indicative of electron acceleration, which is further in line with the hypothesis of the presence of electric fields. However, the detailed mechanism leading to 

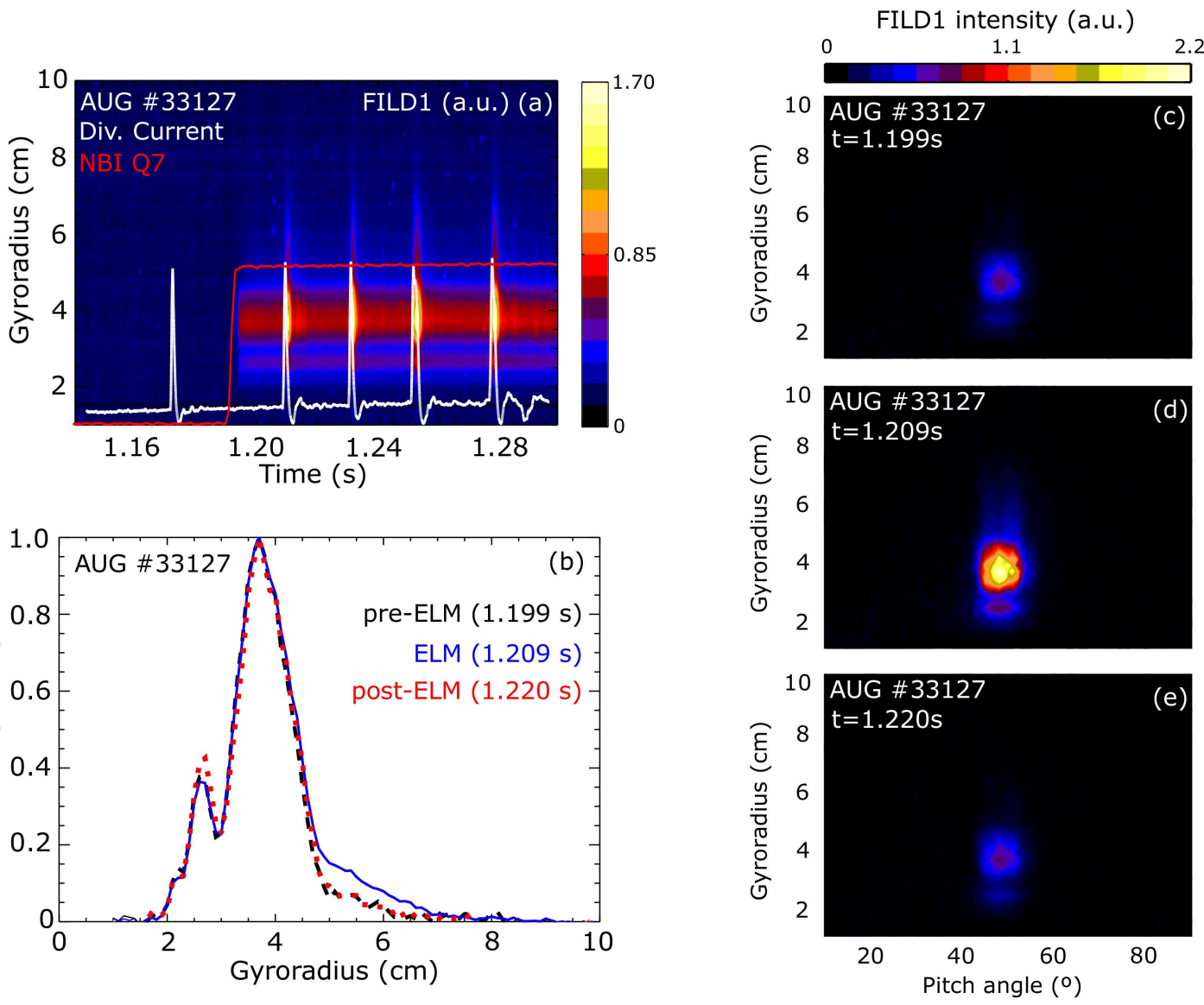

Figure 4. (a) Gyroradius profile evolution of the FILD signal at the relevant pitch angle in AUG shot \#33127. The divertor current (white) is used as an ELM monitor. (b) Comparison between inter-ELM and intra-ELM gyroradii profiles obtained with FILD in shot \#33127. (c), (d) and (e) show the complete velocity space measurement of FILD at these time points.

the electron acceleration and subsequent ECE emission in AUG remains unclear and shall be investigated in detail in the future.

The results of the tomographic inversion together with the variation of the pitch angle structure of the high-energy feature with $q_{95}$, suggest a mechanism for the acceleration of the beam ions which is highly velocity-space dependent. Moreover, the characteristic times associated with the individual fast-ion filaments $(t \sim 100 \mu \mathrm{s})$ in comparison with the fast-ion slowing down time in these plasmas $\left(\tau_{s}=100 \mathrm{~ms}\right)$ indicate that the phenomenon is likely to be collisionless. In view of these observations, a resonant interaction between the beam ions and parallel electric fields emerging during the ELM crash is proposed to be responsible for the acceleration process.

\section{Modelling}

The increased beam-ion losses and accelerated population measured during the ELM with the FILD systems are likely the result of a complex mechanism includ- ing a resonant interaction between the fast-ions and the induced 3D electromagnetic perturbation. Modelling efforts have been carried out in order to gain a deeper insight into this interaction, by performing simulations to investigate separately the effect of magnetic and electric perturbations on fast-ions. The aim of the simulations described here is to qualitatively reproduce the main features of the experimental observations.

\subsection{Magnetic perturbation fields}

Full-orbit following simulations of fast-ions have been performed during a complete ELM crash including realistic magnetic perturbation fields simulated with the 3D non-linear resistive MHD code JOREK [27, 28]. The associated electric fields have not been included at this stage, such that only the deconfinement of fast particles is studied based on JOREK simulations, not their acceleration. No collisions between the fastions and the bulk plasma have been considered in the simulations. In Fig. 8 the magnetic perturbation associated with the ELM is plotted. It can be observed 

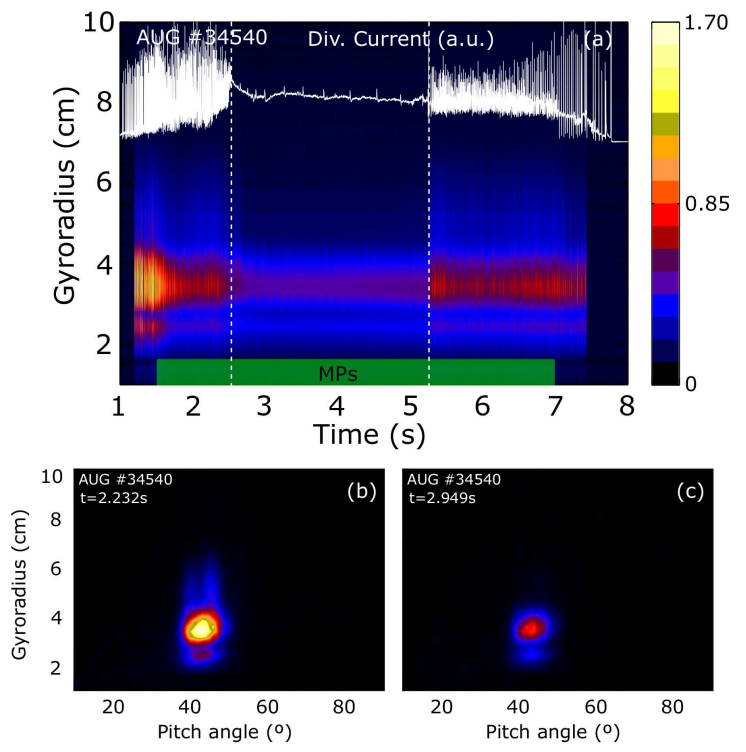

Figure 5. Gyroradius profile evolution of the FILD signal at the relevant pitch angle in AUG shot \#34570, during which external MPs are applied (green box). The time window in which ELM suppression is achieved is indicated by two vertical dashed lines. The complete velocity space measured by FILD is shown at a time point before ELM suppression is achieved (b), and at a time point when ELMs are suppressed (c).

that the perturbation is located at the edge of the plasma. The orbits of the fast-ion losses measured by FILD, which are plotted in white (trapped) and green (passing), explore the region where the perturbation is located. The simulated fast-ion heat load pattern on the AUG wall is shown in Fig.9(a). The footprint located at $\theta=50^{\circ}$ corresponds to the upper edge of the AUG limiters, while the footprint located at $\theta=-100^{\circ}$ corresponds to the lower divertor. The $n=8$ toroidal mode number of the ELM can be observed in the pattern of the losses located around the midplane, which show diagonal structures tilted towards positive increase in the toroidal direction, thus again revealing the 3D nature of the phenomenon. The time evolution of the losses throughout the whole ELM crash is shown in Fig.9(b), where the losses in the entire vessel have been integrated. Several spikes can be observed resembling the filamentary-like pattern of the measured fast-ion losses shown in Fig.1. It should be mentioned that, for computational reasons, the resistivity used in this JOREK simulation is artifitially increased, which might lead to a distortion of the timescale of these losses. The fast-ion loss level in the absence of magnetic perturbation is indicated by a dashed red line for reference.

To further investigate the nature of the fastion transport induced by the ELM perturbation, calculations of the variation of the toroidal canonical momentum $P_{\Phi}=m R v_{\Phi}-Z e \psi$ have been carried out with the orbit following code ASCOT [29]. A set of $40 \cdot 10^{3}$ markers was followed for $500 \mu$ s in a perturbed magnetic configuration within the ELM crash, with a range of initial values in the radial coordinate $R$ and pitch $\Lambda=\frac{v_{\|}}{v}$, defined with respect to the magnetic field, and with a fixed energy of $60 \mathrm{keV}$. In an axisymmetric magnetic configuration, $P_{\Phi}$ is a motion invariant. However, in the presence of 3D symmetry breaking magnetic fields, such as those imposed by the application of external MPs, the variation of the toroidal canonical momentum is a measurement of the radial transport of the ions [30]. An increase in $P_{\Phi}$ implies a decrease in $\psi(Z e>0)$, which corresponds to inwards transport of ions, given that the sign convention used here is such that $\psi$ is negative and, in absolute values, larger at the magnetic axis than at the plasma edge. Oppositely, a decrease in $P_{\Phi}$ corresponds to outwards transport of ions. Fig.10 shows the results of such simulation. The trappedpassing boundary region can be clearly observed. In the region corresponding to the passing orbits, vertical resonant structures are observed, while in the trapped orbits region the resonances show a different pattern.

\subsection{Parallel electric fields}

Simulations including the magnetic field perturbation associated with the ELM qualitatively reproduce the time evolution of the fast-ion losses. However, these cannot account for the observed particle acceleration, for which the presence of an electric field is needed. In order to explore the viability of the proposed acceleration mechanism for the beam ions during ELMs, full orbit particle simulations have been carried out in a realistic magnetic equilibrium for AUG. A simple test model for the parallel electric field, which was kept static during the simulation, was used:

$$
\vec{E}=\overrightarrow{b_{\|}} \cdot A \cdot \exp \frac{\left(\rho-\rho_{0}\right)^{2}}{2 \sigma^{2}} \cdot \cos \left(n \phi-m \theta^{*}+\alpha\right)
$$

where $\overrightarrow{b_{\|}}$is a unit vector parallel to the magnetic field, $A$ is a parameter controlling the amplitude of the electric field, $\rho_{0}$ and $\sigma$ are the centroid and width of the perturbation in minor radius $\rho$, and $n$ and $m$ are the toroidal and poloidal mode numbers respectively. $\phi$ is the toroidal angle coordinate, $\theta^{*}$ the poloidal angle coordinate and $\alpha$ is the phase of the perturbation.

This simple model reproduces the 3D character of the expected parallel electric field associated with the ELM, and its parametrization makes it possible to easily vary its spatial structure and amplitude to perform sensitivity studies. In these simulations a set of $10^{5}$ markers have been followed during $50 \mu \mathrm{s}$, which is the time scale associated with a single fast-ion filament as shown in section 2. A scan was performed 

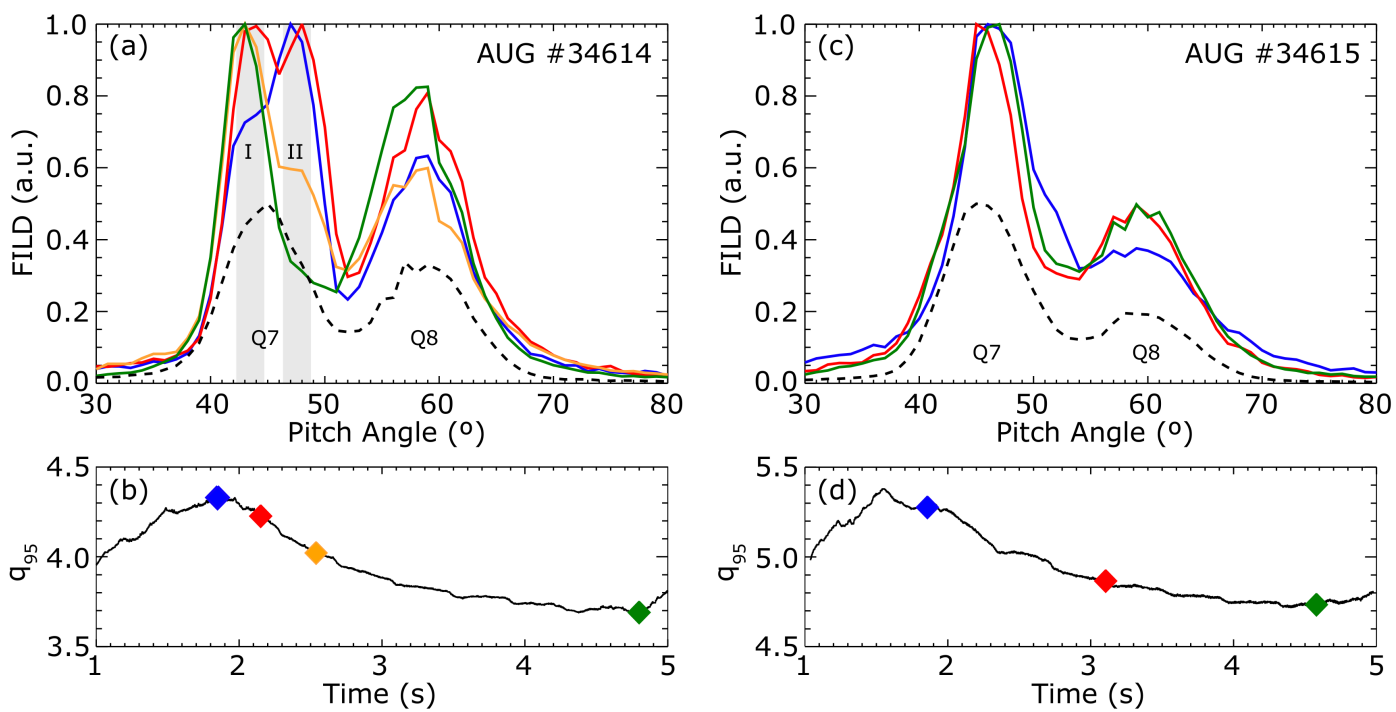

Figure 6. (a) and (c) show the pitch angle profile of the FILD signal for AUG \#34614 and \#34615 respectively. In black, the profile at the main NBI injection energy is plotted for reference (dashed line). The profile at the high-energy component is plotted in different colors for different timepoints indicated in (b) and (d), which shows the evolution of $q_{95}$ with time.
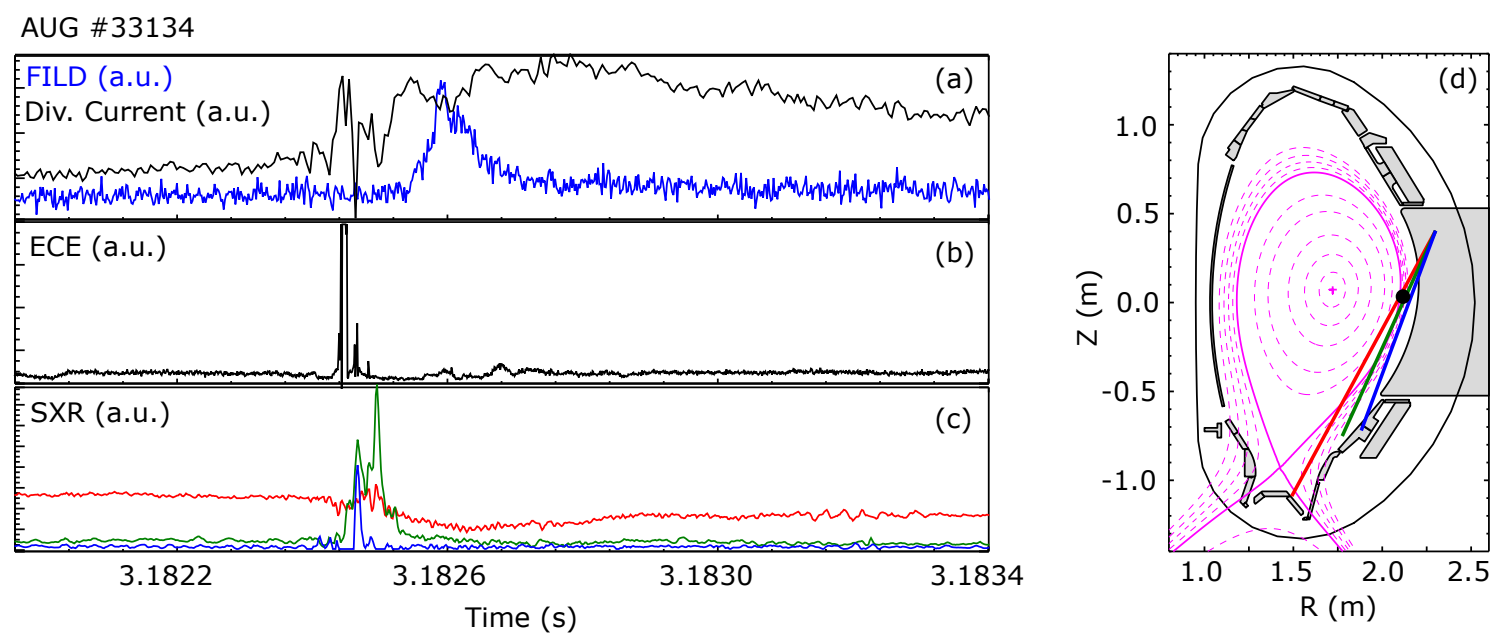

Figure 7. (a) Timetrace of the FILD signal (in blue) and the divertor current (in black) during an ELM in AUG. (b) Timetrace showing a burst in electron cyclotron emission from the plasma edge. (c) Timetraces of three different channels of the soft X-ray diagnostic with lines of sight near the plasma edge. (d) Poloidal plot of the AUG vessel showing in detail the soft X-ray lines of sight and the inferred location of the ECE emission.

in $\mathrm{R}$ and $\Lambda$ with a fixed energy of $90 \mathrm{keV}$. The results are shown in Fig.11, in which the amplitude of the parallel electric field was set to a constant value of $A=2 \mathrm{kV} / \mathrm{m}$, well above the Dreicer electric field for these plasmas. Such large magnitudes for the parallel electric fields were found to appear transiently and locally in non-linear resistive MHD simulations of ELMs, sufficient to explain electron acceleration [8]. A scan in the toroidal mode number of the perturbation was done for $n=10$ (a), $n=7$ (b) and $n=5$ (c), which are in the range of what is expected for ELMs in AUG [31]. The poloidal number was set as $m=q \cdot n$, and the centroid of the perturbation was located in the edge region $\left(\rho_{0}=0.9\right.$ and $\sigma=0.1$ ). It can be observed that only the ions exploring the edge region gain or lose energy. The maximum energy gain obtained is of the order of tens of $\mathrm{keV}$, which is consistent with the experimental observation, and the trend indicates that larger energy gains are obtained for lower toroidal mode numbers. Furthermore, vertical structures can be observed for the ions in the region corresponding to passing orbits $(\Lambda \leq-0.5)$ similar to what is observed in Fig.10. No such clear structures can be observed for the ions in the region corresponding to trapped orbits $(\Lambda \geq-0.5)$. These structures might be explained by linear and non-linear 


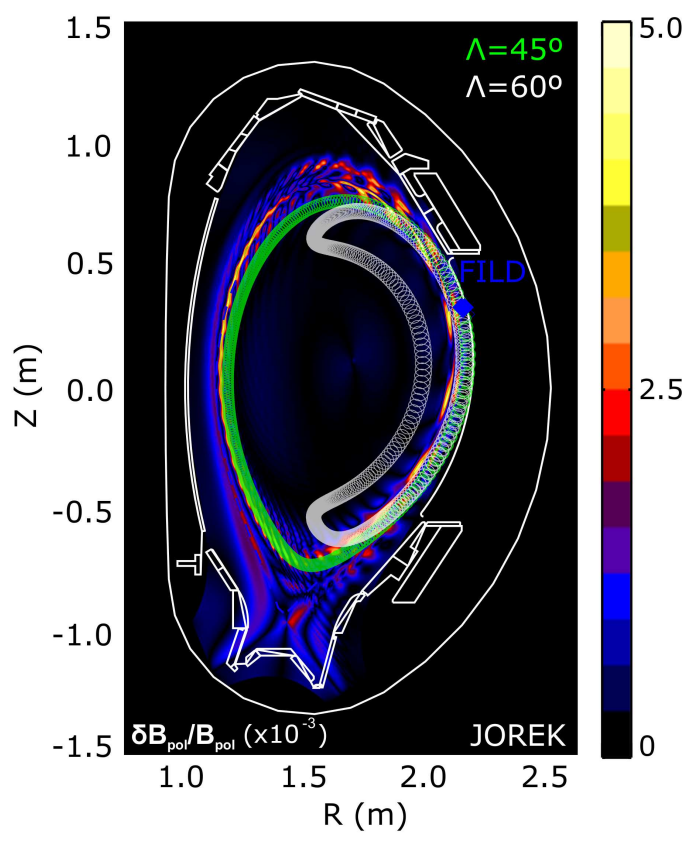

Figure 8. Poloidal contour of the magnetic perturbation of an ELM calculated with JOREK. Typical orbits measured by FILD are overlayed in green and white.

resonances between the particles and the perturbation [32], similar to recent observations in plasmas with externally applied 3D perturbations [30]. The topology of these resonances is reproduced over a wide range of parameters of the perturbation. This is illustrated in Fig.11(d), where the same qualitative pattern is observed for the case in which the perturbation is centered at $\rho_{0}=0.95$ and has a width of $\sigma=0.05$. In Fig.12(a) and (b) the results of a scan in the amplitude of the parallel electric field are shown, where values of $1 \mathrm{kV} / \mathrm{m}$ and $0.1 \mathrm{kV} / \mathrm{m}$ respectively were set. The same patterns are obtained and the maximum energy gain is observed to decrease for lower amplitudes, as expected. These results suggest that the experimentally observed energy gain could be matched by a proper combination of the perturbation parameters such as toroidal mode number, width and amplitude within realistic values.

The FILD experimental measurements are consistent with this picture. The pitch angle structure dependence of the high-energy feature with $q_{95}$ was found to be very clear for passing ions corresponding to beam source Q7, while a less clear dependence was observed for trapped ions corresponding to beam source Q8, as shown in Fig.6. The approximated deposition of beam sources Q7 and Q8 is indicated in Fig.11(a) by two white dashed lines. By ramping $q_{95}$ in the experiment, FILD probes ions with different radial birth positions, and this is equivalent to moving horizontally in Fig.11.
For orbits in the passing region, which are populated by NBI Q7, the structure of the resonances are vertical lines and we would then be jumping from one resonance to another, which is giving rise to the peaks at different pitch angles in the FILD signal. However, for orbits in the trapped region, which is populated by NBI Q8, the structure of the resonances is more complicated and therefore moving along the radial coordinate does not necessarily lead to a different resonance. In this picture, the change in the pitch angle structure of the FILD signal can be understood as the probing of different resonances for different $q_{95}$ values.

The evolution of the velocity space of the markers in the simulation is shown in Fig.13. The plots in the left and right columns correspond to a resonant and non-resonant ion, with an initial pitch of $\Lambda=-0.9$ and initial radial position of $R=2.055 \mathrm{~m}$ and $R=2.140 \mathrm{~m}$ respectively. In Figs.13(a) and (b) the projection of the ion orbit into the poloidal $(\Theta)$ and toroidal $(\Phi)$ angles is overplotted in grey on top of the toroidal electric field at the magnetic surface where the orbit is laying, which is represented by the contour. It can be seen that for the case of the resonant ion (a) the orbit is better aligned with the perturbation than in the case of the non-resonant orbit. Fig.13(c) shows how the resonant ion gains energy continuously while the nonresonant ion (d) does not show a net energy gain. The pitch angle of the resonant ion increases in absolute value, this is, it gains parallel velocity as expected by an acceleration due to a parallel electric field. This is shown in Fig.13(e), where the blue line highlights the pitch angle of the ion at the outer midplane. On the other hand, it can be seen that the pitch angle barely changes for the non-resonant ion (f).

\section{Summary and conclusions}

Intra-ELM time resolved velocity space measurements of fast-ion losses induced by ELMs in the ASDEX Upgrade tokamak have been presented. Multiple fastion filaments, with characteristic times of the order of $100 \mu \mathrm{s}$, are measured with two different toroidally displaced FILD detectors during a single ELM crash, thus revealing the $3 \mathrm{D}$ nature of the phenomenon. The first evidence of beam ion acceleration measured during ELMs has been presented through the observation of a fast-ion population in FILD at energies well above the NBI injection value. These measurements were carried out in plasmas in which the only external source of fastions were the NBI systems, in the absence of ICRH, allowing us to discard accelerated ions of ICRF origin.

The application of tomographic inversion techniques to the FILD signal reveals velocity-space structures in the fast-ion loss distribution during ELMs with unprecendented resolution. The phenomenon is 

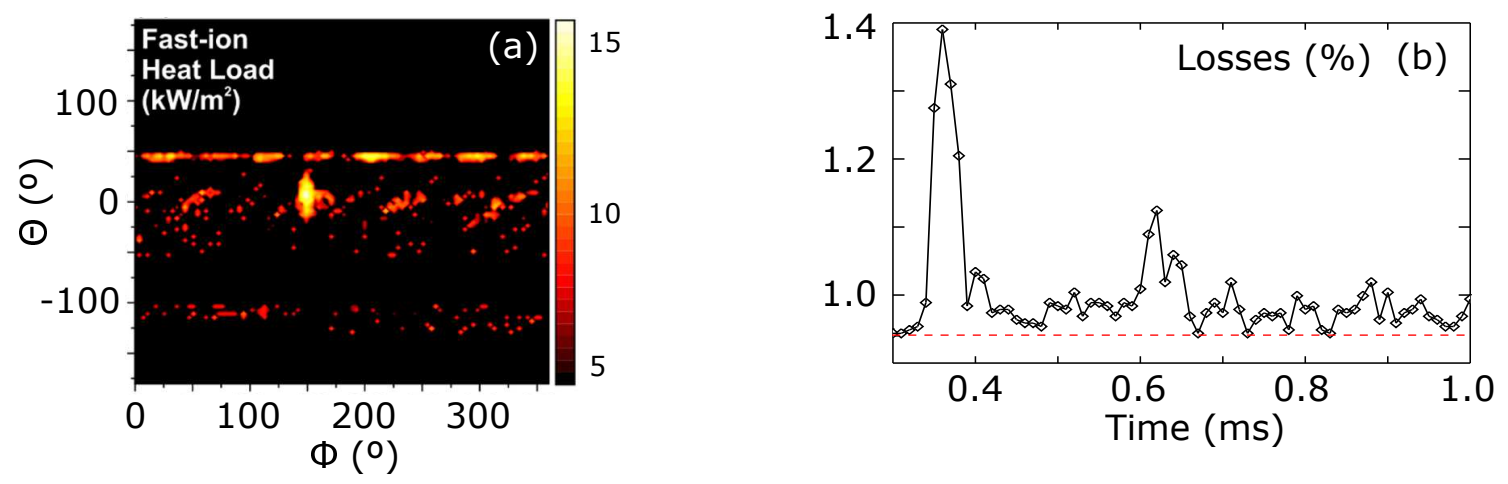

Figure 9. (a) Fast-ion heat load pattern on the AUG walls calculated during a simulated ELM crash with the full-orbit following code Gourdon. (b) Time evolution of the fast-ion losses on the walls during the ELM crash. The dashed red line indicates the level of fast-ion losses in the absence of magnetic perturbation.

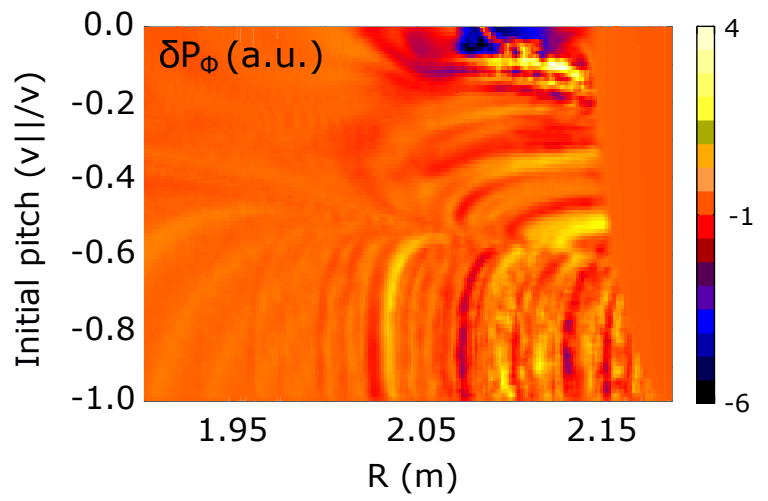

Figure 10. Variation of the toroidal canonical angular momentum calculated with ASCOT during an ELM crash. Positive variation (yellow regions) is interpreted as an inward transport while a negative variation is interpreted as an outward transport (blue regions).

observed in both mitigated and non-mitigated ELM regimes while it is not seen in ELM suppressed regimes. The pitch angle structure of the high-energy feature is observed to depend on the particular NBI source and $q_{95}$. These observations suggest that the acceleration could be explained in terms of a resonant interaction between the fast-ion orbits and the parallel electric field emerging during the ELM, when magnetic reconnection is believed to take place [3]. Measurements of SXR and ECE bursts at the ELM onset, which are indicative of electron acceleration, further support this hypothesis. Future work will focus on parametric studies of the high energy feature with plasma parameters such as collisionality or the main NBI injection energy. In order to investigate differences in the energy gain of the high-energy feature, the tomographic inverted FILD signals need to be compared. For this kind of systematic analysis, improved tomographic methods allowing for faster inversions are required in order to be able to analyse a large number of ELMs in a reasonable computational time.
From the modelling point of view, the main features of the experimental measurements can be retrieved. The filamentary-like pattern of the measured fast-ion losses can be reproduced by performing full-orbit following simulations of fast-ions during a complete ELM crash, including only the magnetic perturbation fields calculated with JOREK. In order to account for the particle acceleration, full-orbit simulations including a parametrized static parallel electric field model have been carried out, in an axisymmetric magnetic equilibrium. This simple model resembles the $3 \mathrm{D}$ structure of the ELM. The results reveal resonant structures in the particles phase space. Consistent with the experimental observations, the topology of these resonances show a clear difference between trapped and passing ions. This is also observed in the variation of $P_{\Phi}$ in simulations including only the ELM magnetic perturbation, which is used as a proxy for radial transport of particles. Energy gains of the order of tens of $\mathrm{keV}$, as observed in the experiments, are obtained in the simulations, assuming toroidal mode numbers which are compatible with experimental observations and parallel electric field amplitudes which were transiently observed in previous modelling capable to explain electron acceleration. However, future work will focus on the modelling of particle acceleration and transport in the presence of time-evolving realistic electromagnetic fields during an ELM, provided by non-linear resistive MHD codes. This will allow to further explore the interaction of fastion orbits with the perturbed electromagnetic fields appearing during the ELM, and how this impacts the resonant interaction between the particles and the perturbation, which could help to gain insights on the proposed acceleration mechanism.

The finding motivates the incorporation of a kinetic description of fast particles in ELM models, and may shed light on the possible contribution of fast-ions to the ELM stability. Additionally, the 

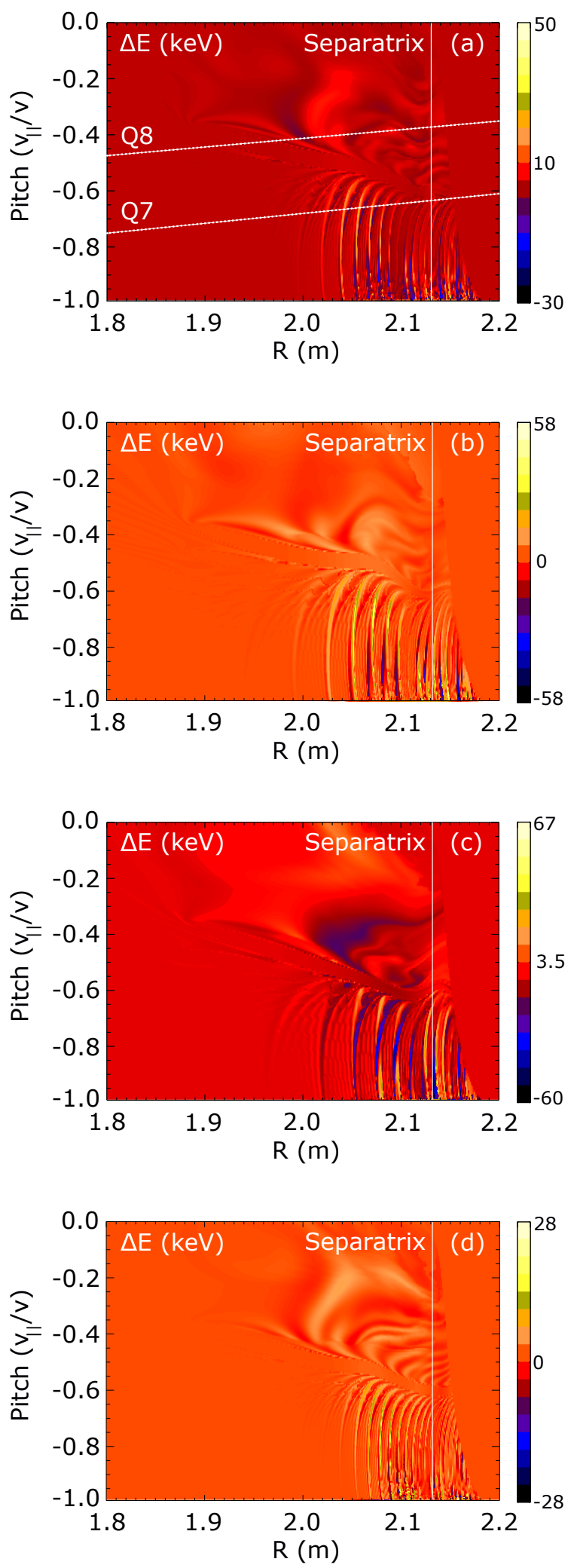

Figure 11. Simulated energy gain of the markers against the initial pitch angle and radial position in the presence of a parallel electric field. The separatrix position is indicated in white for reference. (a) Case with a toroidal mode number of $n=10$, $\rho_{0}=0.9$ and $\sigma=0.1$. The approximated deposition of beams Q7 and Q8 is indicated by the dotted white lines. Cases (b) and (c) are the same as case (a) but with a toroidal mode number $n=7$ and $n=5$ respectively. (d) Case with $n=10, \rho_{0}=0.95$ and $\sigma=0.05$. In all cases similar structures can be observed in the trapped and passing orbit regions. contribution of the observed beam ion acceleration to the overall particle and energy loss during the ELM cycle remains to be investigated for a better understanding of the transient heat loads delivered to plasma facing components and its scaling to future devices. The detailed measurements presented here may also contribute to a better understanding of the fundamental mechanisms underlying particle acceleration in plasmas.

\section{Acknowledgments}

The authors would like to thank M.Hoelzl for very fruitful discussions. Figures 1, 2(a), 3, 4(a), 5(a), $6(\mathrm{a}), 8,9(\mathrm{~b})$ and 11(a) are modified versions of figures included in [33]. This work has been carried out within the framework of the EUROfusion Consortium and has received funding from the Euratom research and training programme 2014-2018 under grant agreement No 633053. The views and opinions expressed herein do not necessarily reflect those of the European Commission. This research was supported in part by the Spanish Ministry of Economy and Competitiveness (Grant Nos. RYC-2011-09152, FIS2015-69362-P, and ENE2012- 31087), the Marie Curie FP7 Integration Grant (No. PCIG11-GA-2012-321455) and the H2020 Marie Sklodowska Curie programme (Grant No. 708257).

\section{References}

[1] H Zohm. Edge localized modes (ELMs). Plasma Phys. Control. Fusion, 38(2):105-128, feb 1996.

[2] F. Wagner, G. Becker, K. Behringer, D. Campbell, A. Eberhagen, W. Engelhardt, G. Fussmann, O. Gehre, J. Gernhardt, G. v. Gierke, G. Haas, M. Huang, F. Karger, M. Keilhacker, O. Klueber, M. Kornherr, K. Lackner, G. Lisitano, G. G. Lister, H. M. Mayer, D. Meisel, E. R. Mueller, H. Murmann, H. Niedermeyer, W. Poschenrieder, H. Rapp, H. Roehr, F. Schneider, G. Siller, E. Speth, A. Staebler, K. H. Steuer, G. Venus, O. Vollmer, and Z. Yu. Regime of improved confinement and high beta in neutral-beam-heated divertor discharges of the ASDEX tokamak. Phys. Rev. Lett., 49(19):14081412 , nov 1982

[3] A. W. Leonard. Edge-localized-modes in tokamaks. Phys. Plasmas, 21(9):090501, sep 2014.

[4] P. B. Snyder, H. R. Wilson, J. R. Ferron, L. L. Lao, A. W. Leonard, T. H. Osborne, A. D. Turnbull, D. Mossessian, M. Murakami, and X. Q. Xu. Edge localized modes and the pedestal: A model based on coupled peelingballooning modes. In Phys. Plasmas, volume 9, pages 2037-2043. American Institute of Physics, may 2002.

[5] R.P. Wenninger, M. Bernert, T. Eich, E. Fable, G. Federici, A. Kallenbach, A. Loarte, C. Lowry, D. McDonald, R. Neu, T. Pütterich, P. Schneider, B. Sieglin, G. Strohmayer, F. Reimold, and M. Wischmeier. DEMO divertor limitations during and in between ELMs. Nucl. Fusion, 54(11):114003, nov 2014.

[6] M. Garcia-Munoz, H. U. Fahrbach, and H. Zohm. Scintillator based detector for fast-ion losses induced by magnetohydrodynamic instabilities in the ASDEX 

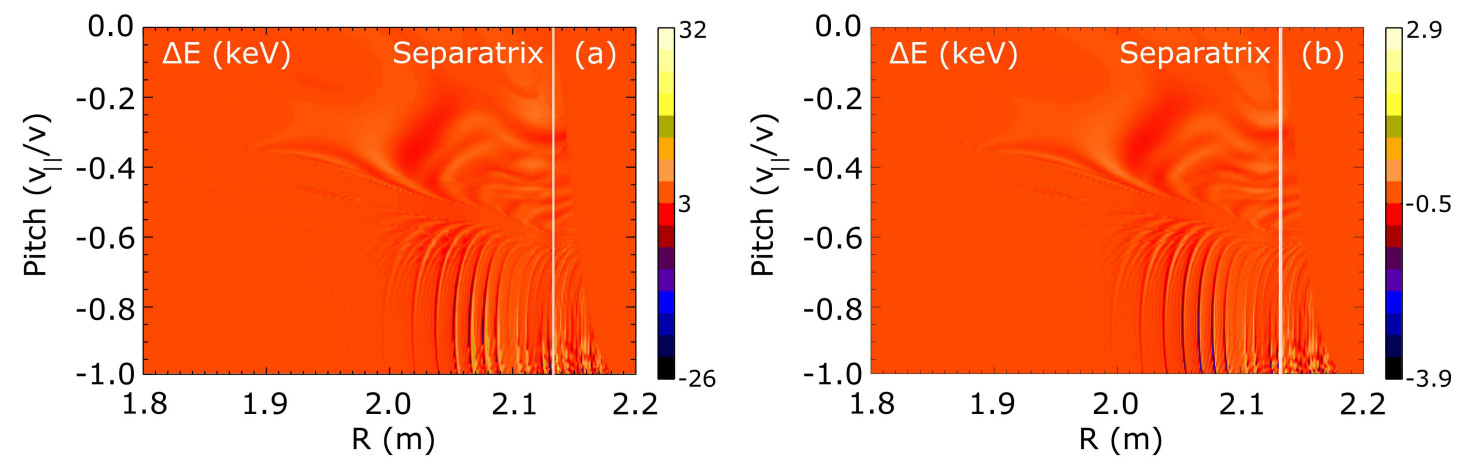

Figure 12. Energy gain of the markers in the presence of parallel electric fields with different amplitudes and $n=10, \rho_{0}=0.9$ and $\sigma=0.1$. (a) $A=1 \mathrm{kV} / \mathrm{m}$. (b) $A=0.1 \mathrm{kV} / \mathrm{m}$.
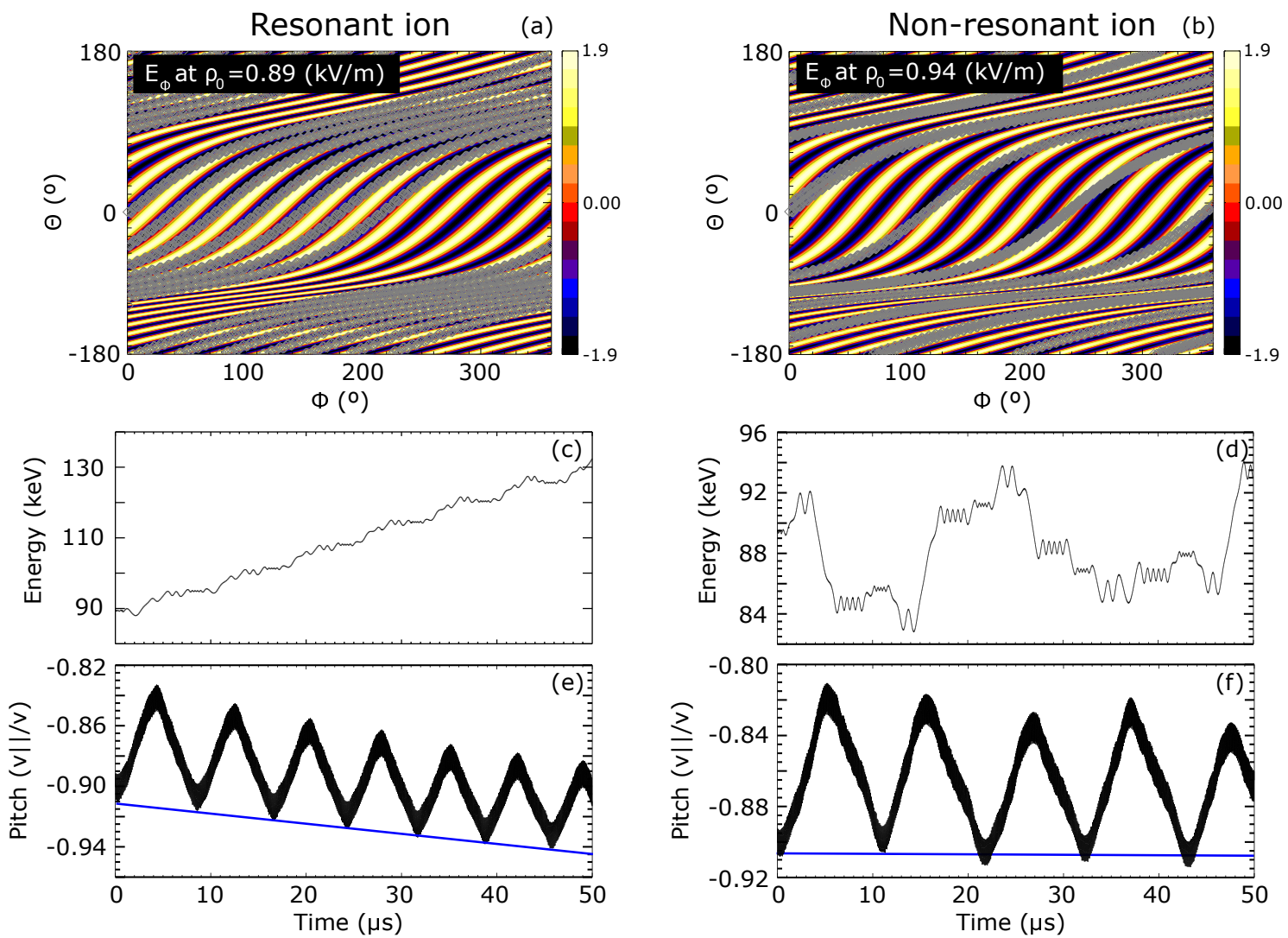

Figure 13. Trajectory (grey) of a resonant (a) and non-resonant (b) ion overplotted on top of the parallel electric field associated with the ELM perturbation. (c) and (d) show the energy evolution of the resonant and non-resonant markers respectively, while (e) and (f) show the corresponding pitch angle evolution. The blue lines show the evolution of the pitch angle at the outer midplane 
upgrade tokamak. Rev. Sci. Instrum., 80(5):053503, may 2009.

[7] Matthias Willensdorfer, Tyler Cote, Michael Thomas Griener, David Anthony Ryan, Erika Strumberger, Wolfgang Suttrop, Nengchao Wang, Marco Cavedon, Severin Sebastian Denk, Mike G Dunne, Rainer Fischer, Joaquin Galdon-Quiroga, Christopher J Ham, Chris C Hegna, Matthias Hoelzl, Andrew Kirk, Marc Maraschek, Alexander Felician Mink, Nils Leuthold, Francois Orain, Egor Seliunin, and Hartmut Zohm. Dynamics of ideal modes and subsequent ELM crashes in 3D tokamak geometry from external magnetic perturbations. Plasma Phys. Control. Fusion, aug 2019.

[8] S. J. Freethy, K. G. McClements, S. C. Chapman, R. O. Dendy, W. N. Lai, S. J.P. Pamela, V. F. Shevchenko, and R. G.L. Vann. Electron kinetics inferred from observations of microwave bursts during edge localized modes in the mega-amp spherical tokamak. Phys. Rev. Lett., 114(12):125004, mar 2015.

[9] P. Helander, L.-G. Eriksson, R. J. Akers, C. Byrom, C. G. Gimblett, and M. R. Tournianski. Ion Acceleration during Reconnection in MAST. Phys. Rev. Lett., 89(23):235002, nov 2002.

[10] S. Eilerman, J. K. Anderson, J. S. Sarff, C. B. Forest, J. A. Reusch, M. D. Nornberg, and J. Kim. Runaway of energetic test ions in a toroidal plasma. Phys. Plasmas, 22(2):020702, feb 2015.

[11] K. G. McClements, J. O. Allen, S. C. Chapman, R. O. Dendy, S. W.A. Irvine, O. Marshall, D. Robb, M. Turnyanskiy, and R. G.L. Vann. Particle acceleration during merging-compression plasma start-up in the Mega Amp Spherical Tokamak. Plasma Phys. Control. Fusion, 60(2):025013, 2018.

[12] K G McClements and M R Turnyanskiy. Energetic particles in laboratory, space and astrophysical plasmas. Plasma Phys. Control. Fusion, 59(1):014012, jan 2017.

[13] G.T.A Huysmans and O Czarny. MHD stability in X-point geometry: simulation of ELMs. Nucl. Fusion, 47(7):659666 , jul 2007.

[14] M Garcia-Munoz, S Äkäslompolo, P. De Marne, M G Dunne, R Dux, T E Evans, N M Ferraro, S Fietz, C Fuchs, B Geiger, A Herrmann, M Hoelzl, B Kurzan, N Lazanyi, R M McDermott, M Nocente, D C Pace, M Rodriguez-Ramos, K Shinohara, E Strumberger, W Suttrop, M A Van Zeeland, E Viezzer, M Willensdorfer, and E Wolfrum. Fast-ion losses induced by ELMs and externally applied magnetic perturbations in the ASDEX Upgrade tokamak. Plasma Phys. Control. Fusion, 55(12):124014, dec 2013.

[15] J. Galdon-Quiroga, M. Garcia-Munoz, M. Salewski, A.S. Jacobsen, L. Sanchis-Sanchez, M. Rodriguez-Ramos, J. Ayllon-Guerola, J. Garcia-Lopez, J. Gonzalez-Martin, M.C. Jimenez-Ramos, J.F. Rivero-Rodriguez, E. Viezzer, and The ASDEX Upgrade Team. Velocity-space sensitivity and tomography of scintillator-based fastion loss detectors. Plasma Phys. Control. Fusion, (60):105005, 2018.

[16] W. W. Heidbrink. Fast-ion D $\alpha$ measurements of the fast-ion distribution (invited). Rev. Sci. Instrum., 81(10):10D727, oct 2010.

[17] M. Salewski, S.K. K. Nielsen, H. Bindslev, V. Furtula, N.N. N. Gorelenkov, S.B. B. Korsholm, F. Leipold, F Meo, P.K. K. Michelsen, D. Moseev, and M. Stejner. On velocity space interrogation regions of fast-ion collective Thomson scattering at ITER. Nucl. Fusion, 51(8):083014, aug 2011.

[18] M. Salewski, B. Geiger, S.K. K. Nielsen, H. Bindslev, M. García-Muñoz, W.W. W. Heidbrink, S.B. B. Korsholm, F. Leipold, F. Meo, P.K. K. Michelsen, D. Moseev, M. Stejner, and G. Tardini. Tomography of fast-ion velocity-space distributions from synthetic CTS and FIDA measurements. Nucl. Fusion, 52(10):103008, oct 2012 .

[19] A S Jacobsen, L Stagner, M Salewski, B Geiger, W W Heidbrink, S B Korsholm, F Leipold, S K Nielsen, J Rasmussen, M Stejner, H Thomsen, M Weiland, and the ASDEX Upgrade Team. Inversion methods for fastion velocity-space tomography in fusion plasmas. Plasma Phys. Control. Fusion, 58(4):045016, apr 2016.

[20] M. Salewski, B. Geiger, A. S. Jacobsen, P. C. Hansen, W. W. Heidbrink, S. B. Korsholm, F. Leipold, J. Madsen, D. Moseev, S. K. Nielsen, M. Nocente, T. Odstrčil, J. Rasmussen, L. Stagner, M. Stejner, M. Weiland, T Odstrcil, J. Rasmussen, L. Stagner, M. Stejner, M. Weiland, and the ASDEX Upgrade team. High-definition velocity-space tomography of fastion dynamics. Nucl. Fusion, 56(10):106024, oct 2016.

[21] Todd E. Evans, Richard A. Moyer, Keith H. Burrell, Max E. Fenstermacher, Ilon Joseph, Anthony W. Leonard, Thomas H. Osborne, Gary D. Porter, Michael J. Schaffer, Philip B. Snyder, Paul R. Thomas, Jonathan G. Watkins, and William P. West. Edge stability and transport control with resonant magnetic perturbations in collisionless tokamak plasmas. Nat. Phys., 2(6):419423, jun 2006.

[22] W. Suttrop, T. Eich, J. C. Fuchs, S. Guenter, A. Janzer, A. Herrmann, A. Kallenbach, P. T. Lang, T. Lunt, M. Maraschek, R. M. McDermott, A. Mlynek, T. Puetterich, M. Rott, T. Vierle, E. Wolfrum, Q. Yu, I. Zammuto, and H. Zohm. First observation of edge localized modes mitigation with resonant and nonresonant magnetic perturbations in ASDEX upgrade. Phys. Rev. Lett., 106(22):225004, jun 2011.

[23] W Suttrop, A Kirk, R Nazikian, N Leuthold, E Strumberger, M Willensdorfer, M Cavedon, M Dunne, R Fischer, S Fietz, J C Fuchs, Y Q Liu, R M McDermott, F Orain, D A Ryan, and E Viezzer. Experimental studies of high-confinement mode plasma response to nonaxisymmetric magnetic perturbations in ASDEX Upgrade. Plasma Phys. Control. Fusion, 59(1):014049, jan 2017.

[24] Maraschek M. Igochine V., Gude A. and ASDEX Upgrade Team. Hotlink based soft x-ray diagnostic on ASDEX Upgrade. Technical report, Max Planck Institute for Plasma Physics, 2010.

[25] M Willensdorfer, S S Denk, E Strumberger, W Suttrop, B Vanovac, D Brida, M Cavedon, I Classen, M Dunne, S Fietz, R Fischer, A Kirk, F M Laggner, Y Q Liu, T Odstrčil, D A Ryan, E Viezzer, H Zohm, and I C Luhmann. Plasma response measurements of external magnetic perturbations using electron cyclotron emission and comparisons to 3D ideal MHD equilibrium. Plasma Phys. Control. Fusion, 58(11):114004, nov 2016.

[26] S.S. Denk, Rainer Fischer, Omar Maj, Emanuele Poli, J.K. Stober, Ulrich Stroth, Branka Vanovac, Wolfgang Suttrop, and Matthias Willensdorfer. Radiation transport modelling for the interpretation of oblique ECE measurements. EPJ Web Conf., 147(02002):02002, jul 2017.

[27] M. Hölzl, S. Günter, R. P. Wenninger, W. C. Müller, G. T.A. Huysmans, K. Lackner, and I. Krebs. Reducedmagnetohydrodynamic simulations of toroidally and poloidally localized edge localized modes. Phys. Plasmas, 19(8):082505, aug 2012.

[28] M. Hoelzl, I. Krebs, A. Lessig, K. Lackner, S. Günter, P. Merkel, R. Wenninger, G. Huysmans, E. Nardon, F. Orain, and the ASDEX Upgrade Team. Nonlinear simulations of edge localized modes and resistive walls. invited talk. In 15th European Fusion Theory Conference (EFTC). Oxford, UK, 2013.

[29] E. Hirvijoki, O. Asunta, T. Koskela, T. Kurki-Suonio, 
J. Miettunen, S. Sipilä, A. Snicker, and S. Äkäslompolo. ASCOT: Solving the kinetic equation of minority particle species in tokamak plasmas. Comput. Phys. Commun., 185(4):1310-1321, 2014

[30] L Sanchis, M Garcia-Munoz, A Snicker, D A Ryan, D Zarzoso, L Chen, J Galdon-Quiroga, M Nocente, J F Rivero-Rodriguez, M Rodriguez-Ramos, W Suttrop, M A Van Zeeland, E Viezzer, M Willensdorfer, F Zonca, and the ASDEX Upgrade Team and the EUROfusion MST1 Team. Characterisation of the fast-ion edge resonant transport layer induced by $3 \mathrm{D}$ perturbative fields in the ASDEX Upgrade tokamak through full orbit simulations. Plasma Phys. Control. Fusion, 61(1):014038, jan 2019.

[31] Felician Mink, Elisabeth Wolfrum, Marc Maraschek, Hartmut Zohm, László Horváth, Florian M Laggner,
Peter Manz, Eleonora Viezzer, and Ulrich Stroth. Toroidal mode number determination of ELM associated phenomena on ASDEX Upgrade. Plasma Phys. Control. Fusion, 58(12):125013, dec 2016.

[32] F Zonca, L Chen, S Briguglio, G Fogaccia, G Vlad, and $\mathrm{X}$ Wang. Nonlinear dynamics of phase space zonal structures and energetic particle physics in fusion plasmas. New J. Phys., 17(1):013052, jan 2015.

[33] J. Galdon-Quiroga, M. Garcia-Munoz, K. G. McClements, M. Nocente, M. Hoelzl, A. S. Jacobsen, F. Orain, J. F. Rivero-Rodriguez, M. Salewski, L. Sanchis-Sanchez, W. Suttrop, and E. Viezzer. Beam-Ion Acceleration during Edge Localized Modes in the ASDEX Upgrade Tokamak. Phys. Rev. Lett., 121(2):025002, jul 2018. 